\title{
Microscopy Study of Carbon Nanotubes Produced by Electrical Discharge in Liquid
}

\author{
V. Manevych ${ }_{* *}^{* *}$, O. Jartovskiy ${ }_{* *}^{*}$ A. Axelevitch ${ }^{* *}$, I. Lapsker ${ }^{* *}$, G. Golan ${ }^{* *}$, G. Makrinich ${ }^{* *}$, B. \\ Gorenstein ${ }^{* *}$, and A. Verdyan \\ * Donbass Engineerig Academy, Kramatorsk, Ukraine \\ ** Holon Institute of Technology, 52 Golomb St., Holon, 58102
}

In this work carbon nanotubes are produced by either electric arc discharges between carbon electrodes or during explosive transition of the hydrocarbon liquids to the gaseous state. The synthesis method described here is restricted to the modified arc discharge technique. Figure 1 presents a principal schematic of the experimental setup. A moving electrode from refractory metal (W, Ta, Fe) was submerged in the liquid alcohol and supplied by DC voltage. The second electrode made from graphite was fixed inside a bath with the ethanol precursor. The discharge was preceded reputably. Disordered spatial nanotubes were deposited on the surfaces of metal electrodes (catalytic transition metals) due to the electrical discharge in the liquid precursor.

The pyrolysis process has been performed at heating speed range $100-250{ }^{0} \mathrm{C} / \mathrm{sec}$ (see Figure 2 ). The hydrocarbon substances such as glycerin and ethanol were studied. To initiate the pyrolysis, the nonorganic catalysts were applied in the quantity no more than $1 \%$ of mass of the organic substance. High rate heating has caused explosive transition of the hydrocarbon substance to the gaseous state. These gases were closed between compressed surfaces providing a high pressure in the closed volume of internal cavity. Combination of high temperatures and high pressure as well as dynamic loads in microvolumes has provided conditions for the formation of spatial carbon structures and carbon-containing substances. Resultant materials in the precipitate form were condensed on the internal surface of the cavity inside the sample.

The deposited on metal electrodes precipitates were studied using the optical microscope and the scanning electron microscope "Stereoscan 430i" equipped with the energy-dispersive analyzer EDX.

Figure 3 presents the microphotography of deposited precipitates obtained on the tungsten electrode with various magnifications. The almost pure surface and the covered surface of the electrode may be seen in figure 3a. The deposit looks like to the coral bush grown on the metal surface along to the longitudinal defects of the electrode surface. A more detail consideration of the coating in figure $3 \mathrm{~b}$ shows the system of twisted silks. A picture in figure 3c represents the spatial disordered tubes system. Figure $3 \mathrm{~d}$ enables distinguish separated spatial continuous nanotubes with average diameter of about $60 \mathrm{~nm}$ and of several micron of length. The spatial location of nanotubes may be explained by consideration of turbulent flows of gaseous products of the alcohol pyrolysis by plasma heating.

Our experiments show the feasibility to create the carbon nanotubes by pyrolysis of liquid alcohol precursors using the plasma discharge submerged in the liquid. It was found that condensed films densities were related with the type and quantity of catalysts. All obtained films contain the spherical carbon microparticles. It was established that spatial carbon structures are formed on the metal clusters. A quantity and type of crystal structures differs depending on the catalysts types. 


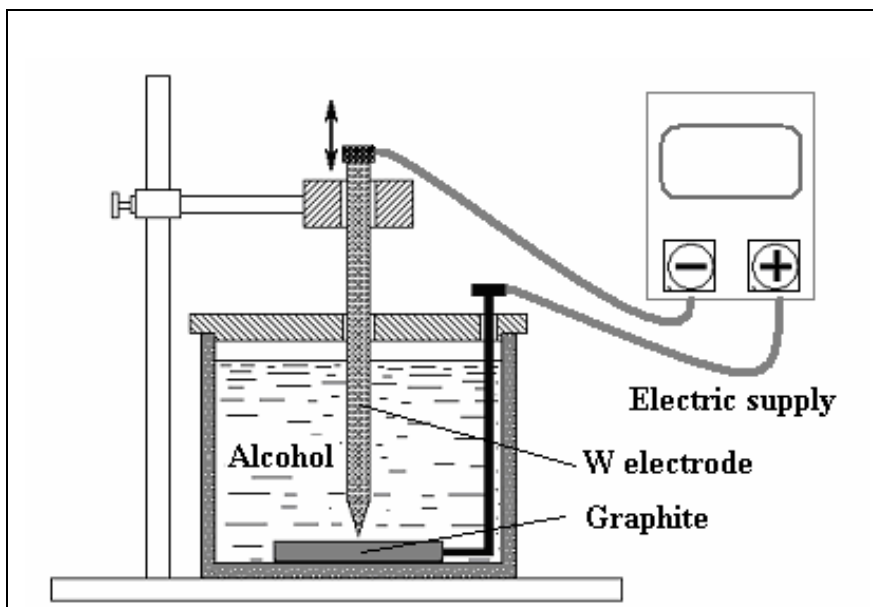

FIG. 1. A principal schematic of the setup for experiments by modified arc discharge technique.

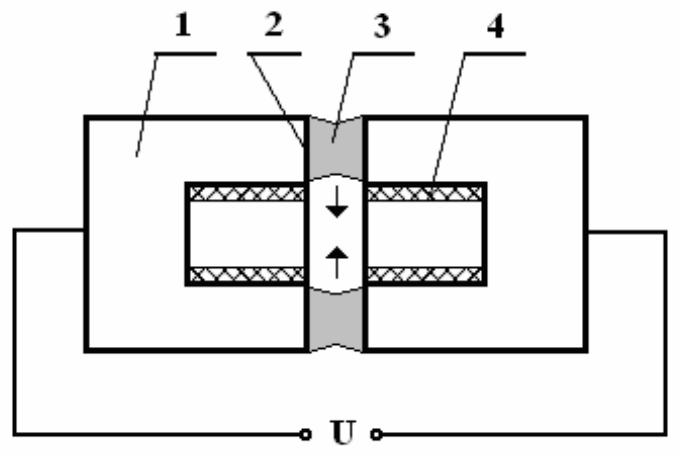

FIG. 2. Principal schematic of the set-up for the hydrocarbon liquids pyrolysis experiments. 1- An experimental sample consists of two parts with the internal cylindrical cavity; 2- Contact surfaces; 3- A hydrocarbon substance; 4- Resultant sediments of the pyrolysis and reactions.
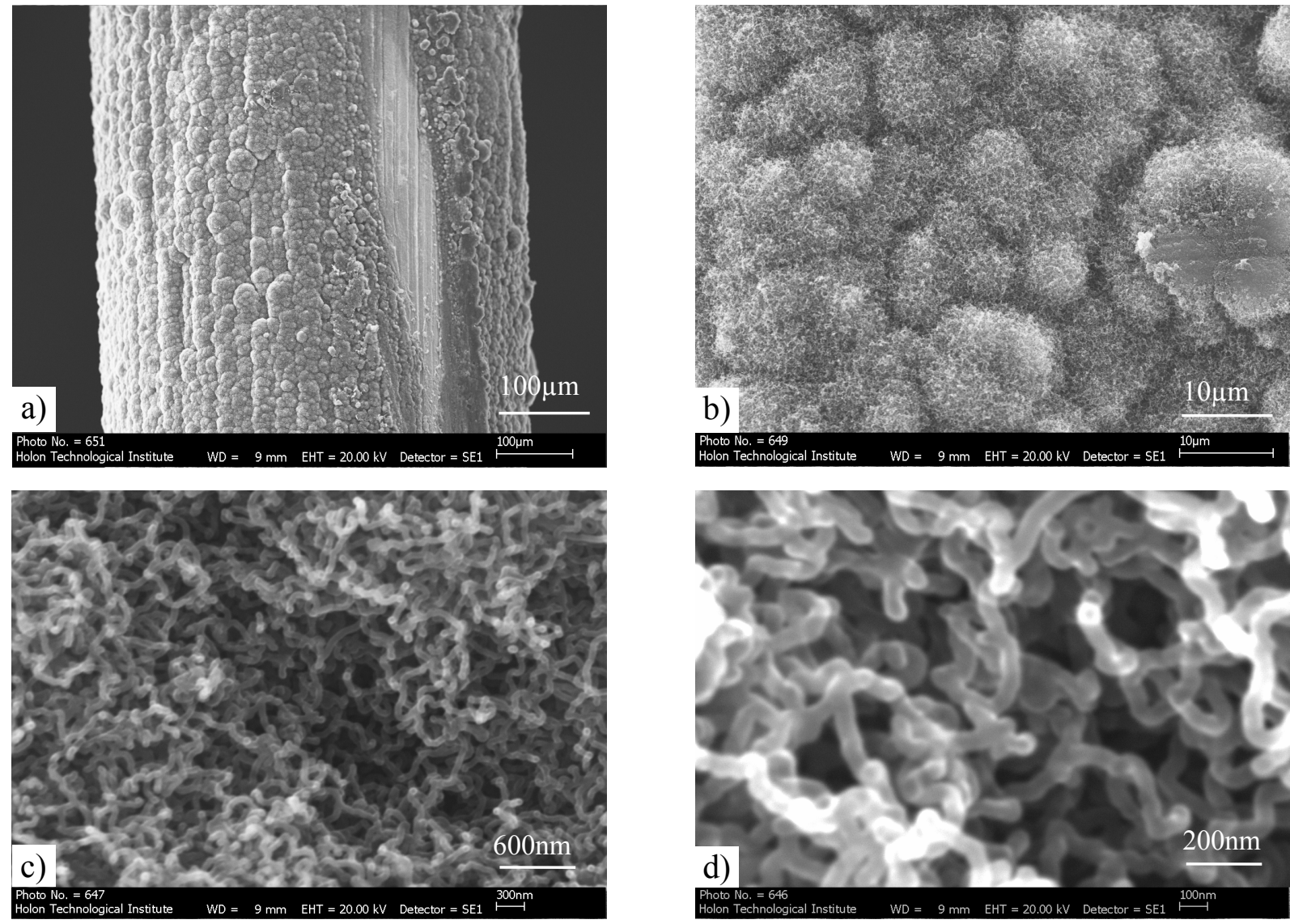

FIG. 3. Microphotography of the deposited spatial nanotubes with various magnifications obtained by scanning electron microscope. 